\title{
Peptide Valency Plays an Important Role in the Activity of a Synthetic Fibrin-Crosslinking Polymer
}

\author{
Robert J. Lamm ${ }^{1}$, Esther B. Lim ${ }^{2}$, Katie M. Weigandt ${ }^{3}$, \\ Lilo D. Pozzo ${ }^{4}$, Nathan J. White ${ }^{2 *}$, Suzie H. Pun ${ }^{1 *}$
}

${ }^{1}$ Department of Bioengineering and Molecular Engineering and Sciences Institute, University of Washington, $372015^{\text {th }}$ Avenue NE, Box 355061, Seattle, WA 98195, USA.

${ }^{2}$ Department of Medicine, Division of Emergency Medicine, University of Washington, Seattle, WA, 98195, USA.

${ }^{3}$ NIST Center for Neutron Research, National Institute of Standards and Technology, Gaithersburg, MD 20899-8562, USA.

${ }^{4}$ Department of Chemical Engineering, University of Washington, Seattle, WA 98195, USA.

*To whom correspondence should be addressed: spun@uw.edu (S.H.P.); whiten4@uw.edu (N.J.W.).

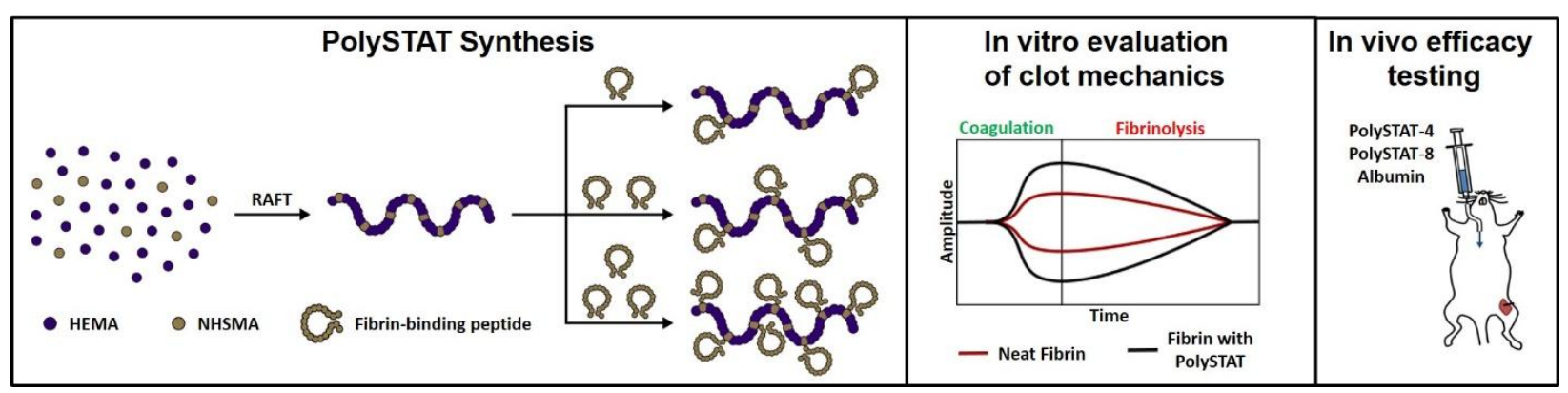

Graphical Abstract 


\begin{abstract}
Therapeutic polymers have the potential to improve the standard of care for hemorrhage, or uncontrolled bleeding, as synthetic hemostats. PolySTAT, a fibrin-crosslinking peptidepolymer conjugate, has the capacity to rescue fibrin clot formation and improve survival in a model of acute traumatic bleeding. PolySTAT consists of a synthetic polymer backbone to which targeting fibrin-binding peptides are linked. For translation of PolySTAT, the optimal valency of peptides must be determined. Grafting of fibrin-binding peptides to the poly(hydroxyethyl methacrylate)-based backbone was controlled to produce peptide valencies ranging from 0 to 10 peptides per polymer. PolySTATs with valencies of $\approx 4$ or greater resulted in increased clot firmness, kinetics, and decreased breakdown as measured by thromboelastometry. A valency of $\approx 4$ increased clot firmness $57 \%$ and decreased clot breakdown $69 \%$ compared to phosphatebuffered saline. This trend was characterized by neutron scattering, which probed the structure of clots formed in the presence of PolySTAT. Finally, PolySTAT with valencies of 4 (100\% survival; $\mathrm{p}=0.013)$ and $8(80 \%$ survival; $\mathrm{p}=0.063)$ improved survival compared to an albumin control in a femoral artery injury model (20\% survival). This work demonstrates tunability of hemostatic polymers and the ability of in vitro assays to predict in vivo efficacy.
\end{abstract}

\title{
Keywords
}

Peptide-Polymer Conjugate, Synthetic Injectable Hemostat, Fibrin-binding Polymer, Ligand Density 


\section{Introduction}

Traumatic injury is the leading cause of death for Americans ages 1 to $44,{ }^{1}$ and hemorrhage, or uncontrolled bleeding, is responsible for $30 \%$ to $40 \%$ of these fatalities. ${ }^{1,2}$ Bleeding is naturally managed through hemostasis, which occurs in two main steps. Primary hemostasis describes the process of quiescent circulating platelets undergoing a morphological and biochemical change upon activation in response to damaged endothelium. Activated platelets express the membrane receptor complex GPIIb/IIIa which binds fibrinogen, resulting in formation of the primary platelet plug. Secondary hemostasis describes the process by which the serine proteases of the clotting cascade undergo a number of activating cleavages. Downstream in the cascade, fibrinogen is cleaved by thrombin to form fibrin, which is able to self-assemble into a fibrous network. Thrombin also activates FXIIIa, which covalently crosslinks the fibrin network resulting in strengthening of the initial platelet plug. The formation of robust clots is critical for stopping blood loss and preventing hemorrhagic shock and death; however, the loss of blood also depletes critical blood proteins necessary for hemostasis. ${ }^{3}$ An additional complication is the development of coagulopathy, or impaired clot formation, that occurs in up to $30 \%$ of trauma patients. Patients with trauma-induced coagulopathy (TIC) form clots that are weaker and have shorter half-lives due to enzymatic clot breakdown by the enzyme plasmin (hyperfibrinolysis), ${ }^{4}$ resulting in 4 to 6 fold increase in mortality when TIC is present upon patient's arrival to the hospital. ${ }^{5,6}$

To combat these effects, human-derived products (whole blood, fresh frozen plasma, and factor concentrates) as well as recombinant factors are used as systemic treatments. While effective, a number of limitations plague these therapies such as short shelf-lives, stringent storage conditions, microbial contamination risks, and the risk of immunogenicity in patients. ${ }^{7}$ 
Synthetic hemostats are therefore in development as an alternative approach to improve clotting function while avoiding the limitations of current treatments.

Several synthetic, platelet-mimicking technologies have been reported to have efficacy in inducing hemostasis in rodent bleeding models. For example, the Lavik group has demonstrated that poly(lactic acid-co-glycolic acid) (PLGA) nanoparticles decorated with platelet-binding RGD moieties decrease bleeding times in animal models of various injuries including sharp, blunt, and blast traumas. ${ }^{8-10}$ Liposome-based platelet-recruiting technologies have also shown success. Okamura et al. showed that adenosine diphosphate-loaded liposomes modified with a platelet-binding $\mathrm{H} 12$ peptide can have procoagulatory effects, decreasing bleeding times in mice and rabbits, ${ }^{11}$ and the Sen Gupta group has reported that multifunctional liposomes that bind von Willebrand factor, collagen, and platelet integrins have hemostatic properties when administered to murine bleeding models. ${ }^{12}$

Synthetic materials targeting secondary hemostasis have also been reported. The Barker group reported a procoagulant microgel formulation that binds fibrin and collapses clot structure based on unique material properties of the microgel. ${ }^{13}$ In addition, our lab recently reported a polymeric hemostat, PolySTAT, that mimics the activity of FXIIIa by physically crosslinking fibrin fibers through its multivalent display of fibrin-binding peptides ${ }^{14}$ (FBPs) along a hydrophilic polymer backbone. ${ }^{15}$ PolySTAT integrates into forming clots, decreases clotting time, and prevents clot lysis while also increasing clot strength. When administered intravenously, PolySTAT also improved survival outcomes in a rat model of femoral artery hemorrhage and fluid resuscitation. Additionally, PolySTAT has been shown to enhance clots in similar fashion to standards of care such as factor concentrates and tranexamic acid. ${ }^{16}$ During initial development, various polymer lengths were investigated, with a degree of polymerization 
of 200 showing optimal clot-enhancing properties using thromboelastography (TEG). Based on the material's performance in vivo, further optimization of the material is being pursued towards ultimate translation.

One previously unexplored property of PolySTAT is FBP valency. When considering targeting ligand display there is a non-obvious balance between potential binding sites, saturating targets, and the effects of ligands on the material structure itself. Notably, multivalency is used to increase binding to target substrates. ${ }^{17}$ Conversely, it has been found in some applications that too high a ligand density can result in decreased binding. ${ }^{18}$ Specifically, ligand density has been shown to play an important role in nanoparticle-based injectable hemostats. ${ }^{19}$ In addition to the efficacy of the material, the peptide valency of the final formulation has significant impact on the

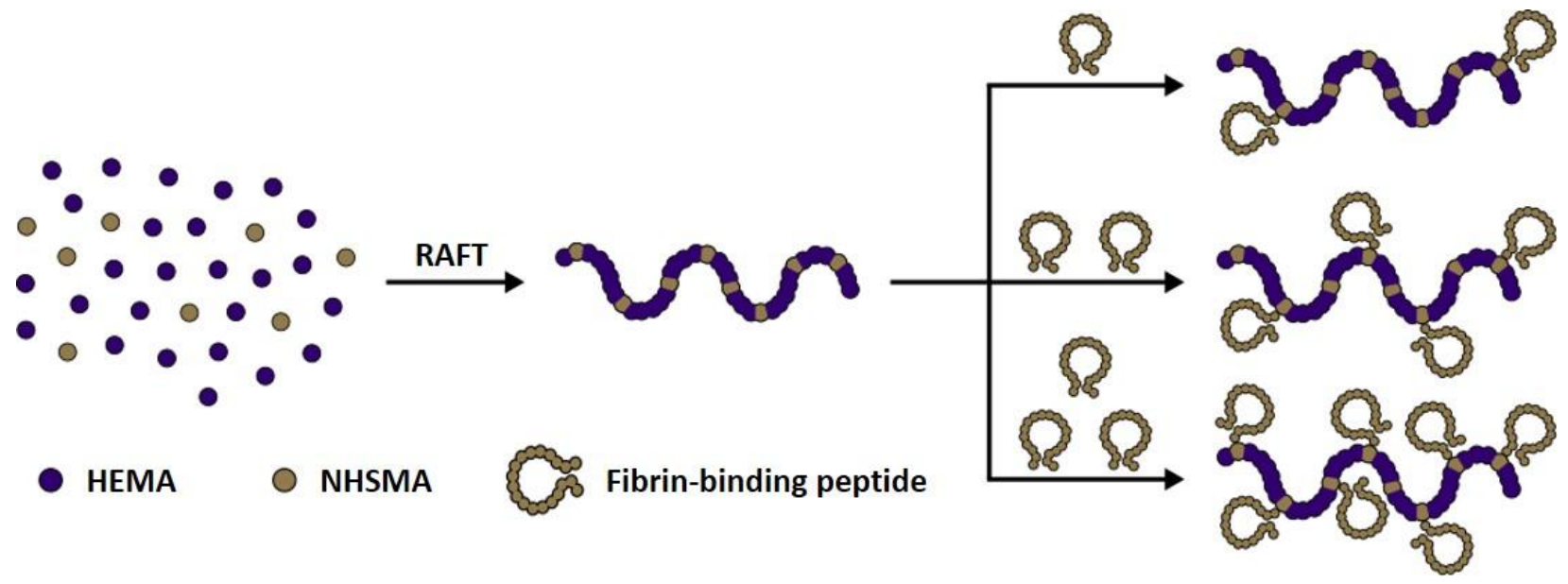

Figure 1. Diagram of synthesis strategy for PolySTAT with varied peptide valencies. Base polymers were synthesized by polymerizing hydroxyethyl methacrylate (HEMA), n-hydroxysuccinimide methacrylate (NHSMA), CTP, and AIBN at an 80:20:1:3 ratio and allowing to react for $24 \mathrm{~h}$ at $70{ }^{\circ} \mathrm{C}$. Fibrin-binding peptide (FBP) was grafted to the backbone at varying ratios of peptide:NHS in presence of organic base and reacted $24 \mathrm{~h}$ at $50{ }^{\circ} \mathrm{C}$.

production costs.

This work reports a structure-function study important for the optimization of PolySTAT composition. PolySTATs with various peptide valencies were synthesized by reversible additionfragmentation chain transfer (RAFT) polymerization followed by peptide grafting (Fig. 1). The 
effect of PolySTAT peptide valency on clot mechanics was determined by rotational thromboelastometry (ROTEM) experiments. High performing valencies were tested to confirm efficacy in a femoral artery and fluid resuscitation model in rats. Finally, neutron scattering, which has been demonstrated as a powerful tool for fibrin structure analysis, ${ }^{20,21}$ was used to probe the effects of PolySTAT with varying peptide valencies on the structure of fibrin gels.

\section{Materials and Methods}

\subsection{Materials}

2,2'-Azobis(2-methylpropionitrile) (AIBN), 4-cyanopentanoic acid dithiobenzoate (CTP), 2-hydroxyethyl methacrylate (HEMA), and all other reagents were purchased from SigmaAldrich (Saint Louis, MO) unless noted otherwise. N-hydroxysuccinimide methacrylate (NHSMA) was purchased from TCI America (Portland, OR). The fibrin binding peptide (FBP; Sequence: Ac-Y(DGl)C(HPr)YGLCYIQGK),${ }^{14}$ developed by the Caravan group, was purchased as a custom order from GL Biochem (Shanghai, China). Thrombin (Fibri-Prest Automate 5) was purchased from Stago (Asnières sur Seine, France). Fibrinogen and plasmin were purchased from Enzyme Research Laboratories (South Bend, IN). Male Sprague-Dawley rats (300 to 360 g) were obtained from Charles River Laboratories (Wilmington, MA). Certain commercial equipment, instruments, or materials are identified in this paper to foster understanding. Such identification does not imply recommendation or endorsement by the National Institute of Standards and Technology, nor does it imply that the materials or equipment identified are necessarily the best available for the purpose.

\subsection{Poly(HEMA-st-NHSMA) synthesis and characterization}

Poly(HEMA-st-NHSMA) was synthesized by reversible addition-fragmentation chain transfer (RAFT) polymerization as described previously. ${ }^{15}$ A final composition of $80 \%$ HEMA 
and degree of polymerization of 200 was targeted by combining HEMA, NHSMA, CTP, and AIBN at a 160:40:1:3 ratio in dimethylacetamide (DMAc) under nitrogen for $24 \mathrm{~h}$ at $70{ }^{\circ} \mathrm{C}$. The desired product was purified from unreacted monomer by $2 \mathrm{x}$ precipitation in diethyl ether, with DMAc as the intermediate solvent, precipitated polymer was collected by centrifugation at 4500 $x$ g. Dithiobenzoate groups were removed via an end capping reaction with 20x molar excess AIBN at $70{ }^{\circ} \mathrm{C}$ for 24 hours. Polymers were characterized via gel permeation chromatography (GPC) with static light scattering and refractive index detectors (MiniDawn Treos and OptilabRex, respectively, both Wyatt Technology, Santa Barbara, CA) to determine molecular weight and polydispersity index (PDI). $\mathrm{H}^{1}$ Nuclear magnetic resonance (NMR) was utilized to determine NHSMA content of the polymer and confirm removal of dithiobenzoate groups.

\subsection{Synthesis of PolySTATs containing varying peptide valencies}

Poly(HEMA-st-NHSMA) was reacted with FBP via the primary amine present in the peptide's lysine residue at the C-terminus under organic basic conditions described by Yanjarappa et $a .^{22}$ Briefly, polymer and peptide were combined with diisopropylethylamine in dimethylsulfoxide and allowed to react at $50{ }^{\circ} \mathrm{C}$ for 24 hours. The molar feed ratios of peptide:NHS were ranged from 0 to 1.5 to target PolySTATs with differing amounts of peptide per polymer. Unreacted NHS groups were then capped with a 10x molar feed of 3aminopropanol. Polymers were purified by extensive dialysis as follows. First, the product was dialyzed against phosphate-buffered saline (PBS) for 24 h (3 buffer changes, 4 L of buffer) during which unreacted peptide precipitates. Contents of the dialysis bag were collected and centrifuged at $4500 \mathrm{x}$ g for 8 min to remove unreacted peptide; the supernatant was collected and moved to a fresh dialysis bag. Dialysis continued for 24 h (3 buffer changes), followed by water for $48 \mathrm{~h}$ (6 dialysate changes) to remove PBS salts. Peptide content of materials was determined 
using the extinction coefficient of FBP and the materials' absorbance at a wavelength of $280 \mathrm{~nm}$ using a NanoDrop 2000 UV-Vis spectrophotometer (Thermo Fisher Scientific, Waltham, MA).

Small-angle x-ray scattering (SAXS) measurements were performed on PolySTATs with varying peptide valency using the standard measurement settings on the SAXS instrument at the Australian Synchrotron ${ }^{23}$. Polymers were prepared in phosphate-buffered saline, loaded into capillaries. Scattering profiles were fitted to the correlation length model, with an additional clustering term, as described by Hammouda $e t a l^{24}$ :

$$
I(Q)=\frac{A}{Q^{n}}+\frac{C}{1+(Q L)^{m}}+B
$$

\subsection{Rotational Thromboelastometry}

ROTEM experiments consisted of $300 \mu \mathrm{L}$ of clotting solution in a standard ROTEM cup placed in a ROTEM whole blood hemostasis analyzer (ROTEM, Basel, Switzerland). Final concentrations in the ROTEM cup were $1.5 \mathrm{mg} / \mathrm{mL}$ fibrinogen, $0.5 \mathrm{IU} / \mathrm{mL}$ thrombin, $4 \mu \mathrm{g} / \mathrm{mL}$ plasmin, $0.1 \mathrm{mmol} / \mathrm{L} \mathrm{CaCl}_{2}$, and 5 or $20 \mu \mathrm{mol} / \mathrm{L}$ PolySTAT. Measured parameters in ROTEM include: (i) the clotting time (CT), measured as the time between reagent addition to clot formation; (ii) $\alpha$-angle, which reflects the rate of clot formation, (iii) the maximum clot firmness (MCF), the highest strength observed for the clot, (iv) the lysis index-30 minutes (LI-30), the percentage of MCF retained 30 minutes after initiation of clot formation, and (v) maximum lysis (ML), the percentage of clot strength lost compared to the MCF at the end of analysis.

\subsection{PolySTAT evaluation in a rat femoral artery injury and fluid resuscitation model}

Animal use was carried out in accordance with protocols approved by the University of Washington Institutional Animal Care and Use Committee. The rat femoral artery injury and fluid resuscitation model was performed as described previously. ${ }^{15}$ Sprague-Dawley rats 
weighing 300-360 g were randomized into one of three treatment groups (albumin, PolySTAT-4, and PolySTAT-8). Rats were anesthetized with isoflurane followed by ketamine-xylazine cocktail injection in the hindlimb. Catheters were placed in the carotid artery and jugular vein for blood pressure measurement and administration of polymers, respectively. Blood gas and metabolites were measured to maintain healthy baseline respiration and lactate levels (carbon dioxide, $\leq 55 \mathrm{~mm} \mathrm{Hg} ; \mathrm{O}_{2}$ saturation, $\geq 95 \%$; lactate, $\leq 1.0 \mathrm{mM}$ ).

After baseline blood gas and metabolites were confirmed appropriate, the femoral artery in the left hindlimb was identified and isolated. Microsurgical clamps were placed at the proximal and distal ends of the isolated vessel to prevent bleeding while a $3 \mathrm{~mm}$ longitudinal incision was made using microscissors. Once the injury was made, a controlled catheter bleed was completed to ensure that all animals started at a normalized mean arterial pressure of 40-50 mm Hg. Clamps were removed and the treatment injected immediately. Depending on treatment group, albumin, PolySTAT-4, or PolySTAT-8 solution $(10 \mathrm{mg} / \mathrm{mL})$ were injected over $1.5 \mathrm{~min}$ at a dose of $15 \mathrm{mg} / \mathrm{kg}$.

For the first 15 minutes, animals bled freely from the femoral artery wound. After $t=15$, animals received saline infusion at a rate of $3 \mathrm{~mL} / \mathrm{min}$ per kilogram to restore mean arterial pressure to at least $60 \mathrm{~mm} \mathrm{Hg}$ up to a total of $60 \mathrm{~mL} / \mathrm{kg}$.

Blood loss over time was measured by collection of blood at 5 minute intervals in preweighed gauze by the blinded researcher at the wound edges with care not to disturb the femoral artery or the clot.

\subsection{Neutron scattering characterization}

Samples for small-angle neutron scattering (SANS) and ultra-small-angle neutron scattering (USANS) were prepared as described previously. ${ }^{20}$ Briefly, deuterated water 
containing 99.9\% $\mathrm{D}_{2} \mathrm{O}$ obtained from Cambridge Isotope Laboratories (Andover, MA) was used to prepare PBS containing $0.5 \mathrm{~mol} / \mathrm{L} \mathrm{NaCl}$. Fibrinogen was dialyzed against the deuterated buffer. Stock PolySTAT solutions were prepared in deuterated buffer as well. Fibrinogen solutions were degassed under vacuum to prevent signal-altering bubbles. $0.5 \mathrm{IU} / \mathrm{mL}$ of thrombin was added to each sample, mixed, and immediately loaded it into the SANS or USANS cells in the presence of $5 \mu \mathrm{mol} / \mathrm{L}$ PolySTAT or a volume control of deuterated PBS.

Small-angle neutron scattering (SANS) and ultra-small-angle neutron scattering (USANS) measurements were performed on the same samples at the Center for Neutron Research at NIST in Gaithersburg, Maryland as described previously. ${ }^{20}$ SANS measurements were performed on the NG7 30-meter instrument. ${ }^{25}$ Measurements were performed at detector distances of $1.3 \mathrm{~m}, 7 \mathrm{~m}$, and $13.2 \mathrm{~m}$ to allow for a q range of $0.002 \AA^{-1}$ to $0.3 \AA^{-1}$. Neutron wavelengths were $5 \AA$ and $8.4 \AA$. All SANS data were corrected for background and empty cell scattering. Data were measured on an absolute scale relative to flux of the direct beam. ${ }^{26}$ USANS measurements were performed on the BT5 perfect crystal diffractometer. ${ }^{27}$ All SANS data were reduced and desmeared using the NIST macros in Igor. ${ }^{26}$

Data were then analyzed using previously established methods for fibrin gels. ${ }^{20} \mathrm{~A}$ modified Guinier fit for one-dimensional structures was used to determine the cross-sectional fiber radius of fibrin fibers within the protein gels. Moreover, a fractal model was used to measure the correlation length and fractal dimension of the fractal structure corresponding to branching of fibrin fibers within the protein gels. ${ }^{20}$

The Guinier fit was performed on the region that includes the turnover to low- $q$ and the intermediate Porod region $\left(0.001 \AA^{-1}<q<0.01 \AA^{-1}\right)$. The intensity and $q$ in the Guinier region are related to the cross-sectional radius of gyration by the following equation: ${ }^{28}$ 


$$
(q I)_{Q \rightarrow 0}=(q I)_{0} \exp \left(-\left(\frac{R_{g}^{2}}{2}\right) q^{2}\right)
$$

where $q$ is scattering vector, $I$ is scattering intensity, and $R_{g}$ is the cross-sectional radius of gyration. Using this approach, an average radius of gyration is determined and the assuming cylindrical structure of fibrin fibers, as previously, the fiber radius can be determined by the following equation: ${ }^{20,28}$

$$
R=R_{g} \sqrt{2}
$$

where $R$ is the true radius of the fiber and $R_{g}$ is the cross-sectional radius of gyration from the Guinier fit. The fiber radius was found to increase with increasing peptide valency (Table 2).

The network structure was modeled using a fractal model that utilizes spherical building blocks based on the work of Teixeira. ${ }^{29}$ The data from the USANS range $\left(q<0.001 \AA^{-1}\right)$ was fitted through least-squares to the fractal described by the following three equations: ${ }^{29}$

$$
I(q)=P(q) S(q)
$$

where $I(q)$ is the total intensity, which is made up of the form factor, $P(q)$, and the structure factor, $S(q)$. The form factor is described as: ${ }^{29}$

$$
P(q)=\varphi V_{P}^{2} \Delta \rho^{2}\left(\frac{\sin \left(q R_{0}\right)-q R_{0} \cos \left(q R_{0}\right)}{3\left(q R_{0}\right)^{3}}\right)^{2}
$$

where $\varphi$ is the volume fraction of spherical building blocks, $V_{P}$ is the volume of the spheres, $\Delta \rho$ is the difference in scattering length density, $q$ is the scattering vector, and $R_{0}$ is the sphere radius. The structure factor is described as: ${ }^{29}$

$$
S(q)=1+\frac{\sin \left[\left(D_{f}-1\right) \tan ^{-1}(q \xi)\right]}{\left(q R_{0}\right)^{D_{f}}} \frac{D_{f} \Gamma\left(D_{f}-1\right)}{\left[1+1 /\left(q^{2} \xi^{2}\right)\right]^{\left(D_{f}-1\right) / 2}}
$$


where $D_{f}$ is the fractal dimension and $\xi$ is the correlation length. The difference in scattering length density was determined through measurements performed by Weigandt et al. ${ }^{20}$ The radius of the spheres was defined as the radius found from the Guinier model. During fitting, $D_{f}$, $\xi$, and $\varphi$ were allowed to vary with while minimizing $\chi^{2}$.

\section{Results}

\subsection{Synthesis and characterization of PolySTATs with varying peptide valencies}

PolySTATs with varying peptide valencies

were synthesized by first preparing a backbone polymer, poly(HEMA-st-NHSMA), by RAFT polymerization followed by peptide grafting and conversion of unreacted NHSMA to alcohols. All
Table 1. Variation of Peptide Valencies

\begin{tabular}{|c|c|c|}
\hline $\begin{array}{c}\text { Peptide:NHS } \\
\text { Molar Feed }\end{array}$ & \multicolumn{1}{c}{$\begin{array}{c}\text { Peptides } \\
\text { per Polymer }\end{array}$} & Title \\
\hline 1.5 & 10.4 & PolySTAT-10 \\
\hline 1.0 & 7.9 & PolySTAT-8 \\
\hline 0.5 & 4.2 & PolySTAT-4 \\
\hline 0.25 & 2.9 & PolySTAT-3 \\
\hline 0.1 & 1.4 & PolySTAT-1 \\
\hline 0.0 & 0.3 & PolySTAT-0 \\
\hline
\end{tabular}

polymers were synthesized using the same backbone polymer to ensure that the degree of polymerization of all materials is held constant. Polymers were synthesized with molecular weight $(31.7 \mathrm{kDa})$ and NHSMA content (19\%) similar to theoretical values $(28.2 \mathrm{kDa}$ and $20 \%)$ as confirmed by GPC and ${ }^{1} \mathrm{H}$ NMR, respectively. Degree of polymerization was 225 .

Polymers with increasing FBP

valencies were obtained by increasing peptide feed ratios during peptide grafting. The peptide valency, in average peptides per polymer, for the various reaction feeds are shown in Table 1. Polymer conjugates with average peptide content of 0 to 10

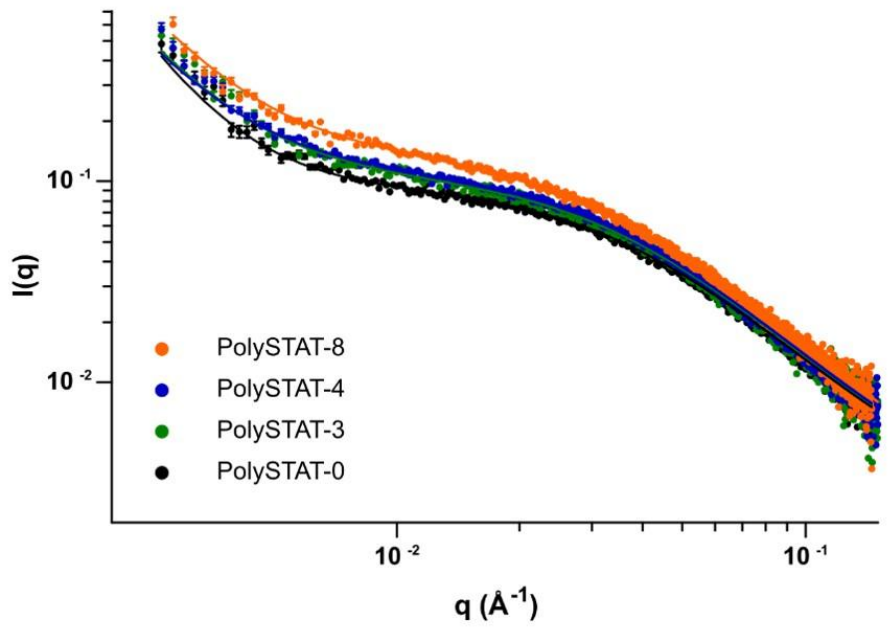

Figure 2. X-ray scattering of PolySTAT with various peptide valencies. PolySTATs were dissolved in phosphatebuffered saline. Measurements were taken using the SAXS instrument at the Australian Synchrotron. Experimental data are displayed as averages as points with uncertainty from reduction as error bars that are often hidden behind data points and fit lines. Correlation length model fits for each condition are shown as solid lines 
peptides per polymer were synthesized. The pHEMA backbone and PolySTATs with a range of peptide valencies showed no lysis of red blood cells at $25 \mu \mathrm{M}$ concentrations suggesting hemocompatibility at approximately 5-fold greater than the administered in vivo dose of 15 $\mathrm{mg} / \mathrm{kg}(5.2 \mu \mathrm{M})$ (Fig. S1).

SAXS measurements and fits (Fig. 2) demonstrated that with an increase in peptide content, the radius of gyration Table 2. PolySTAT radii of gyration of the polymer-peptide conjugate increased (Table 2). Additionally, an increase in low-q scattering intensity was observed for all samples. This is likely due to clustering effects

\begin{tabular}{|c|c|}
\hline Sample & Rg $(\mathbf{\AA})$ \\
\hline PolySTAT-8 & 115.9 \\
\hline PolySTAT-4 & 105.0 \\
\hline PolySTAT-3 & 104.7 \\
\hline PolySTAT-0 & 97.1 \\
\hline
\end{tabular}
of the pHEMA backbone of the material.

Clustering of water-soluble polymers due to hydrophobic interactions has been observed to give similar low-q features to those observed in these meaurements ${ }^{24,30}$.

\subsection{In vitro evaluation of PolySTATs by ROTEM}

Rotational thromboelastometry (ROTEM), a clinically used method to characterize coagulation, was used to determine the effect of polymers on clot mechanics. Two PolySTAT concentrations tested, $5 \mu \mathrm{mol} / \mathrm{L}$ and $20 \mu \mathrm{mol} / \mathrm{L}$, were used. The lower $5 \mu \mathrm{M}$ concentration mimics the conditions of the previous PolySTAT studies and corresponds to $\approx 15 \mathrm{mg} / \mathrm{kg}$ in vivo dose for the various polymers. The higher $20 \mu \mathrm{M}$ concentration corresponds to about two PolySTAT polymers per potential binding site on fibrin. A distinct threshold of peptide valency necessary for PolySTAT to impact clot half-life or formation rate was observed (Fig. 3A, 3B). Polymers with fewer than an average of 3 peptides/polymer did not impact LI-30 or $\alpha$-angle; in contrast, polymers with greater than an average of 4 peptides/polymer showed significantly increased kinetics of clot formation and delay in clot breakdown. It is interesting to note that at a 
higher concentration of PolySTAT $(20 \mu \mathrm{mol} / \mathrm{L})$, the kinetics of clot formation are slightly decreased for the clots formed in the presence of PolySTATs with more than 4 peptides per polymer on average. Thus, the threshold peptide valency required for clot strengthening activity by ROTEM was determined to be $\approx 4$ peptides per polymer on average.

Based on these results, the effect of $5 \mu \mathrm{M}$ PolySTAT-4 on clot mechanics was fully tested against a PBS volume control. Fig. 3C-E show the effects of PolySTAT-4 on CT, MCF and ML. There was a decrease, though not statistically significant, in the CT in the presence of PolySTAT-4 at $5 \mu \mathrm{M}(\mathrm{p}=0.086)$. The MCF was increased $57 \%(12.7 \mathrm{~mm}$ to $20.0 \mathrm{~mm}, \mathrm{p}=$ 0.007), indicating stronger clot formation in the presence of PolySTAT-4 (Fig. 3D). The ML at 50 minutes was decreased 69\% (79.3\% to $24.3 \%, \mathrm{p}<0.001)$ for fibrin clots formed with PolySTAT-4, confirming the antifibrinolytic activity of PolySTAT. 

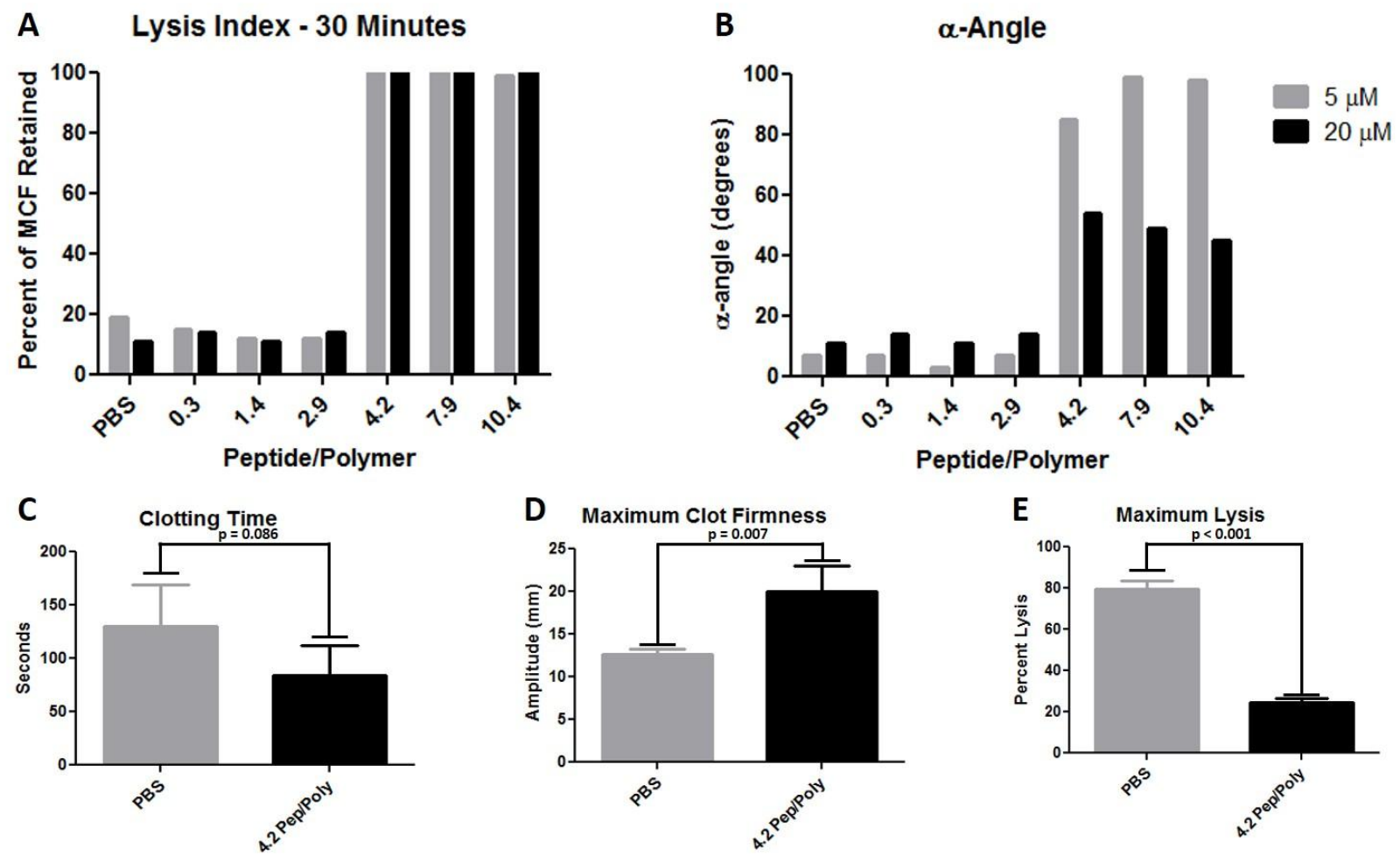

Figure 3. In vitro characterization of fibrin clot kinetics, clot strength, and fibrinolysis. The effect of PolySTAT with various peptide valencies was tested in a hyperfibrinolytic model using ROTEM. The amount of clot firmness retained 30 minutes after clot initiation (A) and the clot kinetics (B) were tested for all peptide valencies at concentrations of $5 \mu \mathrm{M}$ PolySTAT and $20 \mu \mathrm{M}$ PolySTAT $(n=1)$. (C-E) Clotting time, clot strength, and total fibrinolysis of fibrin clots in presence of either a PBS control or the PolySTAT variant containing 4.2 peptides per polymer. Data are averages \pm standard deviation $(n=3) . P$ values were determined using the student's t-test.

\subsection{In vivo performance of PolySTATs in femoral artery injury and fluid resuscitation model}

In the previously reported development and characterization of PolySTAT, rats treated with PolySTAT-16 in a femoral artery and fluid resuscitation model showed significantly improved survival over scrambled peptide controls, an oncotic control (albumin), a volume control (saline), and the body's natural fibrin crosslinker hFXIIIa. ${ }^{15}$ Here, PolySTATs with average valencies of 4 and 8 were found to also be effective in comparison to animals treated with albumin as an osmotic control (Fig. 4B). Animals in the albumin group showed 20\% survival over the entire model with deaths occurring within the first twenty minutes. All animals 
A
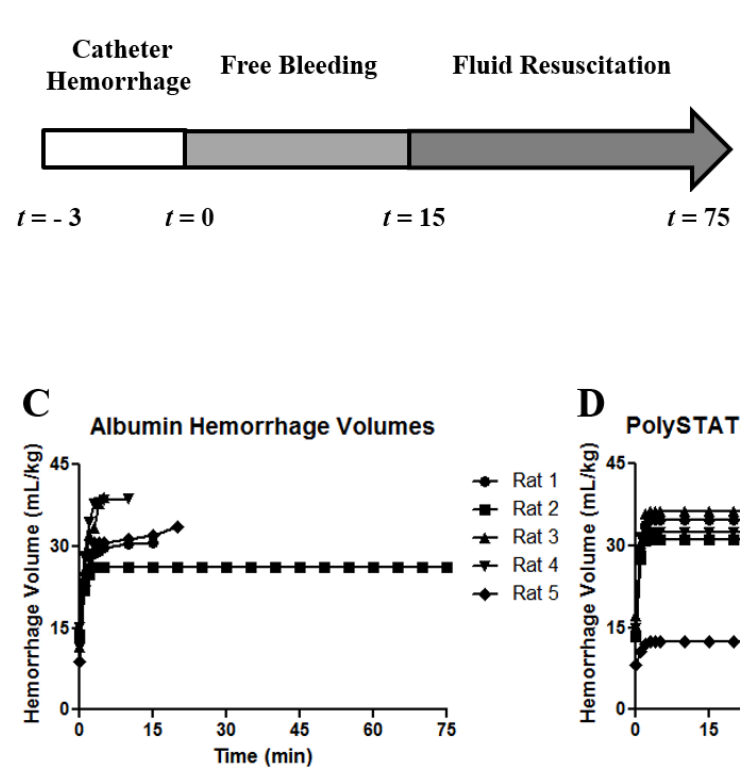

B

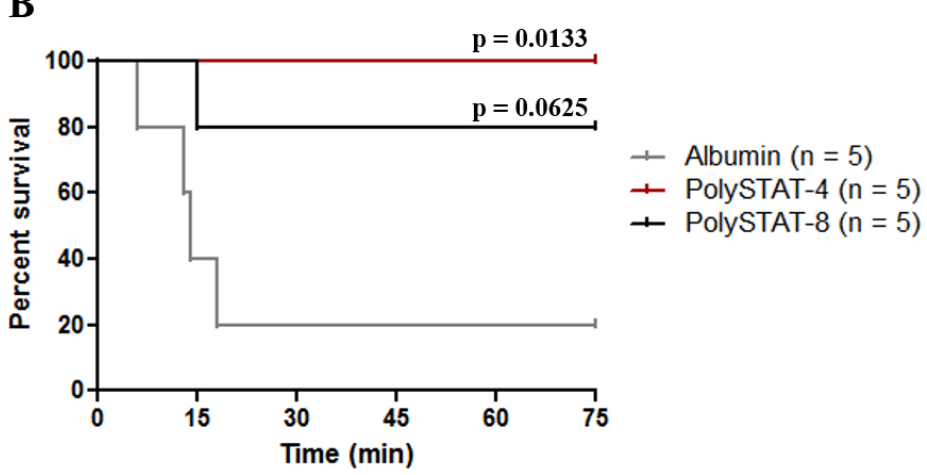

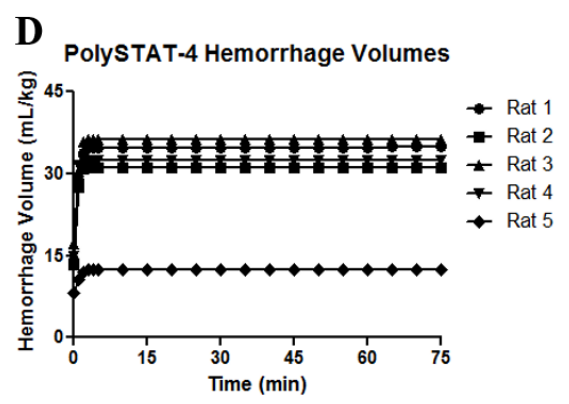

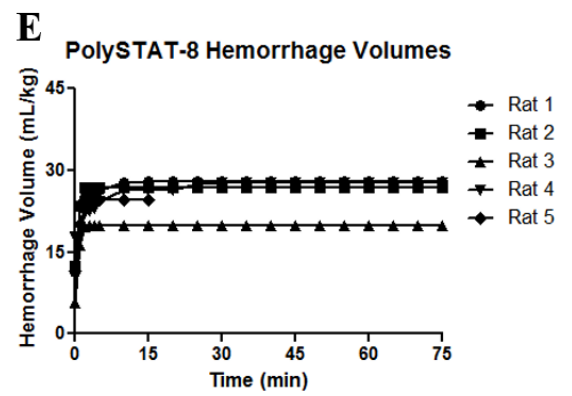

Figure 4. In vivo evaluation of PolySTAT with different valencies in a rat traumatic injury model. (A) Outline of the experiment. Rats underwent catheter hemorrhage to normalize starting blood pressures. Subsequently, clamps proximal and distal to the wound were removed and the treatment was injected. The injury was allowed to bleed freely for 15 minutes, at which point fluid resuscitation began in the form of $0.9 \%$ saline infusion. (B) Survival of animals over 75minute experiment ( $\mathrm{n}=5$ per condition). $P$ values determined by log-rank Mantel-Cox test with comparisons against albumin control. (C-E). Cumulative hemorrhage volumes for individual animals in each condition.

treated with PolySTAT-4 survived through the 75-minute model; this translates to a statistically significant increase in survival in comparison to albumin $(\mathrm{p}=0.013)$. Of the 5 rats treated with PolySTAT-8, a single animal did not survive, resulting in $80 \%$ survival, a non-significant increase in survival in comparison to albumin-treated animals $(p=0.063)$. Hemorrhage volumes were measured for each animal and are reported as milliliter of blood lost per kilogram (mL/kg). Animals treated with albumin (Fig. 4C) generally did not clot well at the injury site, demonstrated by a lack of plateau in blood loss. The exception is the lone surviving rat that clotted and kept its clot strength throughout the experiment. All rats treated with PolySTAT-4 exhibited clotting with no rebleeding events (Fig. 4D). All rats treated with PolySTAT-8 clotted within 15 minutes. There was one minor rebleeding event. Notably, the animal that did not 
survive did not appear to hemorrhage greatly, but was deemed a clinical death with a prolonged mean arterial blood less than $20 \mathrm{~mm} \mathrm{Hg}$.

\subsection{Neutron scattering measurements of fibrin gels with PolySTAT}

Neutron scattering was used to determine the effect of PolySTAT on fibrin structure. Fibrin clots were formed in the presence of PolySTATs with peptide valencies of approximately $0,2,4$, and 8. Despite full solubility at $10 \mathrm{mg} / \mathrm{mL}$ in $\mathrm{H}_{2} \mathrm{O}$ PBS, PolySTAT did not fully dissolve in the deuterated buffer used for neutron scattering studies, therefore the soluble fraction of PolySTAT was collected as supernatant after centrifugation and used. These samples were characterized by UV spectroscopy to determine peptide content and polymer concentration, which are reported in Supplementary Table 3. Due to the hydrophobic nature of FBP, polymer populations with lower peptide grafting ratios resulted in higher solubility (Table S3). Thus, the average peptide valency of polymers dissolved in $\mathrm{D}_{2} \mathrm{O}$ was different from the parent populations. The true peptide valency is used to label the conditions in Figure 5. 
Samples were prepared in uniform fashion with degassed solutions in SANS and USANS sample cells. Scattering data were fully reduced, desmeared, and combined into a single scattering profile (Fig. 5). Signals for neat fibrinogen and PolySTAT-0 appear similar throughout the entire q range. Additionally, PolySTAT-2, -4 , and -8 overlap with these samples throughout

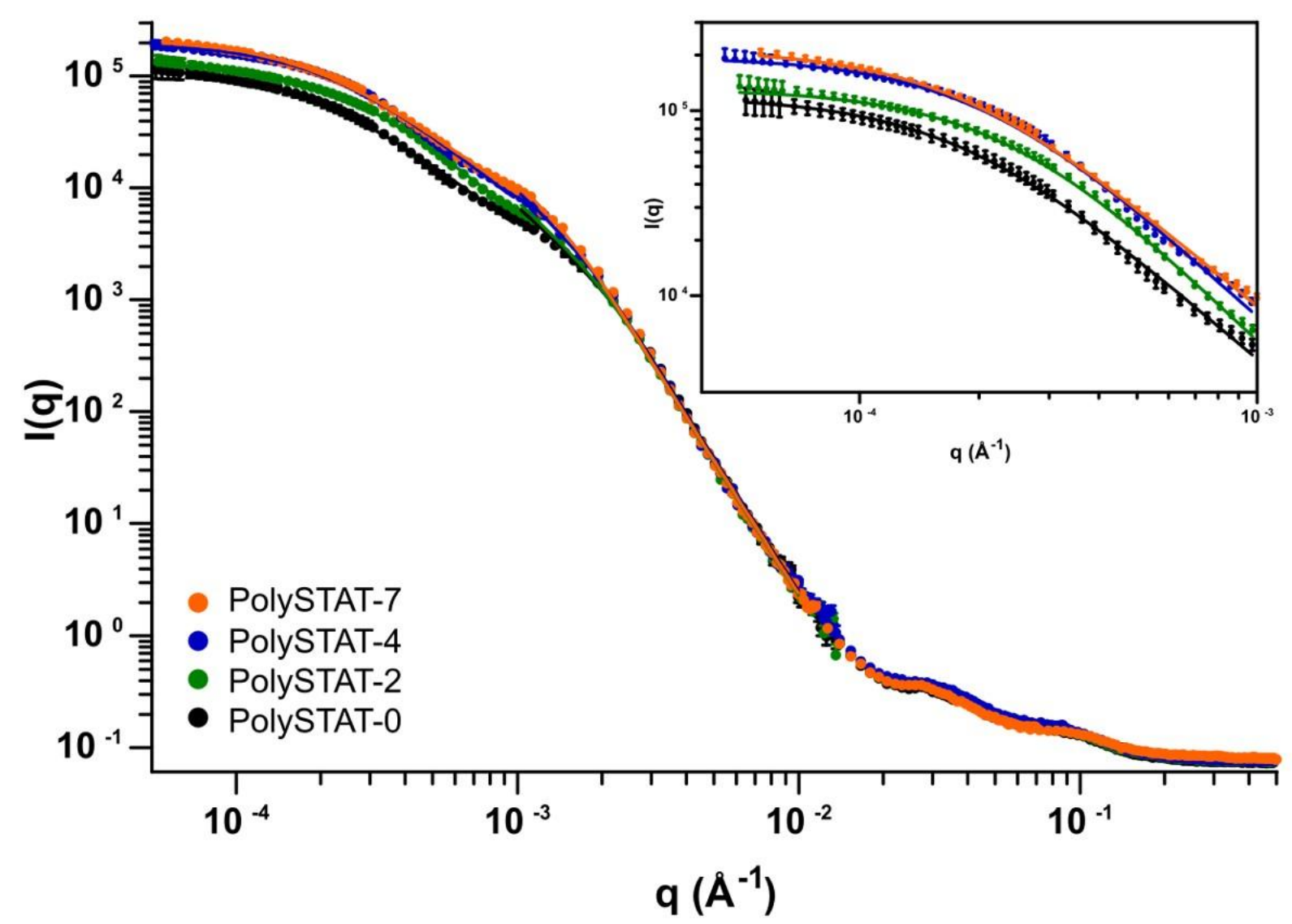

Figure 5. Neutron scattering of fibrin gels with various PolySTATs. Fibrin gels were formed in deuterated solvent by activation with thrombin in the presence of PolySTAT variants. Measurements were taken on SANS and USANS instruments. The data were desmeared and combined into a single scattering profile per sample. Experimental data are displayed as averages points with error bars from the reduction that are often hidden behind data points and fit lines. Guinier model fits for each condition are shown as solid lines for the region $\left(0.008<q<0.01 \AA^{-1}\right)$. Fractal model fits for various conditions are shown as solid lines for the region $(0.00005$ $<0.008 \AA^{-1}$ ). Inset, Fractal model fits and data within the fractal model range. 
the SANS regime of high- and mid-q. However, there is a change in scattering intensity for PolySTAT with 8 and 4 peptides/polymer in the

low-q USANS regime. Using strategies

Table 3 Guinier model fit results: fiber radius

previously used to analyze neutron scattering

data of fibrin gels, a modified Guinier fit for one-dimensional structures was used to determine the cross-sectional fiber radius

of fibrin fibers within the protein gels. Moreover,

a fractal model was to measure the correlation

length and fractal dimension of the fractal

\begin{tabular}{|c|c|c|}
\hline Sample & $\mathbf{R}(\mathbf{n m})$ & $\mathbf{R}(\mathbf{n m})$ \\
\hline PolySTAT-0 & $34.1 \pm 0.4$ & $48.2 \pm 0.6$ \\
\hline PolySTAT-2 & $37.9 \pm 0.5$ & $53.6 \pm 0.7$ \\
\hline PolySTAT-4 & $41.7 \pm 0.5$ & $59.0 \pm 0.7$ \\
\hline PolySTAT-7 & $46.9 \pm 0.6$ & $66.3 \pm 0.8$ \\
\hline
\end{tabular}

structure corresponding to branching of fibrin fibers within the protein gels. ${ }^{20}$

The correlation length describes the

distance over which the fractal dimension is

accurate. From the fits, both the correlation

length and the fractal dimension have no distinct

trend with $\xi$ ranging from 281 to $529 \mathrm{~nm}$ and $D_{f}$
Table 4. Fractal model fit results: fractal structure

\begin{tabular}{|c|c|c|}
\hline Sample & $\begin{array}{c}\text { Correlation } \\
\text { Length }(\mathbf{n m})\end{array}$ & $\begin{array}{c}\text { Fractal } \\
\text { Dimension }\end{array}$ \\
\hline PolySTAT-0 & $529.4 \pm 31.3$ & $1.92 \pm 0.05$ \\
\hline PolySTAT-2 & $381.1 \pm 18.3$ & $2.21 \pm 0.05$ \\
\hline PolySTAT-4 & $451.1 \pm 18.8$ & $2.11 \pm 0.04$ \\
\hline PolySTAT-7 & $519.6 \pm 22.2$ & $1.99 \pm 0.03$ \\
\hline
\end{tabular}

ranging from 1.92 to 2.21 (Table 4).

\section{Discussion}

While synthetic materials that mimic the effects of platelets have seen success, there is currently little work creating synthetic materials mimicking other components of clot formation. Our initial design of a FXIIIa-inspired polymeric hemostat that physically crosslinks fibrin consisted of a polyHEMA backbone with an average of 16 FBP grafted per polymer. ${ }^{15}$ In this initial work, effects of polymer length were investigated, but not the amount of peptide per polymer. Varying the peptide valency could potentially vastly alter the interaction between 
PolySTAT and the fibrin clot. Increasing peptide valency could allow for increased fibrin crosslinking; however, there is likely a saturation point at which increasing peptide content does not increase the positive effect of PolySTAT. Additionally, increased peptide content could lead to instability of the conjugate as the peptide is highly hydrophobic. Furthermore, minimizing the peptide content per polymer without sacrificing efficacy would aide translation to clinical use.

To investigate the effect of peptide valency on PolySTAT efficacy for hemostasis, PolySTAT polymers were synthesized with varying amounts of peptide per polymer. First, a poly(HEMA-st-NHSMA) backbone was synthesized, controlling the degree of polymerization and NHSMA content via the monomer:CTA and HEMA:NHSMA ratios, respectively. PolySTATs with various FBP valencies ranging from 0 to 10 were then synthesized from the poly(HEMA-st-NHSMA) backbone by grafting FBP to the polymer at various mole ratios of peptide: polymer. Importantly, this reaction is reproducible and scalable. Larger batches of PolySTAT-4 and PolySTAT-8 with similar polymer properties were prepared for animal studies (SI Tables S1 \& S2).

ROTEM of fibrin clots formed under lytic conditions with the various PolySTATs showed a threshold of biological activity at $\approx 4$ peptides per polymer. It was not surprising that polymer backbones lacking peptides had no effect on clotting. It was also predictable that conjugates with valencies of $\approx 2$ did not influence clotting, as these lack the ability to bind the fibrin network in a multivalent fashion in independent locations. These results demonstrate the importance of including greater than 2 binding ligands in cross-linking materials. This agrees with the observation by Soon et al. that 4-arm PEG decorated with 4 fibrin knob-mimetic glycine-proline-arginine-proline (GPRP) peptides significantly increased fibrin chain- 
crosslinking compared to a similarly decorated bifunctional PEG with 2 GPRP peptides conjugated. $^{32}$

Another potential explanation for enhanced clotting with increased peptide valency is due to the increased amphiphile character due to incorporation of the hydrophobic FBP. Amphiphilic materials have been shown to promote hemostasis through non-specific interactions. ${ }^{31}$ However, previous work with PolySTAT compared it against PolySCRAM, which substitutes FBP with a scrambled peptide sequence. This scrambled sequence retains the peptide's highly hydrophobic nature while changing the amino acid sequence which ablates specific binding to fibrin. As PolySCRAM performed similar to PBS controls in vitro and in vivo, amphiphilicity has little to no contribution to PolySTAT's activity.

It is also interesting to note that in vitro results shown here provide possible insight into the unknown binding site of FBP. FBP was developed through initial phage display discovery followed by engineering for stability and binding affinity, ${ }^{14}$ although the exact binding site to fibrin was not determined. GPRP peptides, as knob mimetics, are known to interact with fibrin at the knob-hole interactions. ${ }^{32,33}$ Despite similar crosslinking activity of GPRP-PEG and PolySTAT, the functional outcome for GPRP-PEG and PolySTAT are very different. While PolySTAT at different valencies can increase fibrin gel strength, decrease clotting time, and strongly decrease fibrinolysis, ${ }^{15}$ 4-arm GPRP-PEG had minimal effects of clotting time and fibrinolysis, and decreased fibrin gel elastic modulus due to GPRP peptides binding at a site which disrupts natural fibrin polymerization. ${ }^{32}$ These data suggest that FBP does not interact nor interfere with the knob-hole interactions that are integral to fibrin self-assembly.

This hypothesis is further supported by the results from the neutron scattering experiments. Probing the high- to mid-q range correlates to investigating the fibrin monomer to 
individual coarse fiber length scale. ${ }^{20}$ Nearly identical scattering signals for all samples throughout this range suggests little to no deviation in internal fiber structure of the fibrin gel in respect to the neat fibrin gel (Fig. 5).

The difference in scattering signals were observed in the mid- to low-q regimes, and through fitting it was determined that the greatest effects were at the fiber radius level. The radius of fibrin fibers was found to increase with increasing peptide valency, and trended linearly with peptide valency with an $\mathrm{R}^{2}$ of 0.9992 (Supplemental S5). This may be due to the greater PolySTAT valencies leading to the recruitment of additional fibrin, or adding to the mass of individual fibers. It is worth noting that in these experiments the fiber radii are similar to those observed by Weigandt et al but systematically smaller. ${ }^{20}$ Finally, the fits related to the network structure showed no consistent difference in the network. The correlation length and fractal dimension were similar to those observed for neat fibrin by Weigandt $e t a l .^{20}$ This suggests that the changes PolySTAT incurs on the fibrin gel is primarily at the individual fiber length scale. These results give insight into the mechanism by which PolySTAT promotes clotting, suggesting that PolySTAT conducts its effects on the fiber structure of fibrin gels, and also the potential binding site for FBP. A possible explanation for the observation that PolySTAT does not affect fibrin gel structure at small length scales despite its presence at the onset of fibrinogen activation could be that FBP binds at regions where fibrin monomers have self-assembled, perhaps overlapping two or more fibrin monomers.

ROTEM characterization of clot mechanics revealed that PolySTAT with a valency greater or equal to 4 peptides per polymer $(4,8$, and 10) showed enhanced clot properties in contrast to PolySTAT with valencies lower than 4 (0, 1, and 3). Similarly, Shoffstall et al showed that for their platelet-mimicking RGD-modified PLGA nanoparticles, an increased number of 
RGD ligands presented by PLGA nanoparticles resulted in a lower concentration necessary to promote clotting in a liver trauma model. At increased concentrations, we observed a saturating effect on clot kinetics (alpha angle) by the high valency PolySTATs. This is likely due to saturation of binding sites in the early steps of clot formation when fibrin is being generated by thrombin's cleavage of fibrinogen. This observation agrees with those by Shoffstall et al. that high concentrations of particles with high ligand densities resulted in a loss of procoagulatory effects. ${ }^{19}$

Previously, ROTEM characterization has been a good predictor of in vivo success for injectable hemostats. ${ }^{9,15}$ Furthermore, the Lavik group's ligand density work provided evidence for ROTEM as a method for screening different formulations of hemostatic materials. ${ }^{19}$ The results in this study further support ROTEM-based prediction for in vivo success in femoral artery bleeding models. Intravenous administration of both PolySTAT-4 and PolySTAT-8 improved survival rates over 75 minutes in the rat femoral artery injury model. An endpoint of 75 minutes was chosen to allow ample time for rebleeding to occur. In our previous work with the model, all rebleeds began before 30 minutes post-injury, ${ }^{15}$ and the same was observed in these studies. Investigation of hemorrhage rate of the animals suggests that animals treated with PolySTAT-4 did tend to lose more blood (Fig. 4D \& 4E, S4A). This is especially true when focusing on the 4 very similar animals in each treatment group. For both treatments, however, it appears that blood loss ceased quickly and contributed to survival. Notably, the animal that did not survive in the PolySTAT-8 group did not show greater hemorrhage than its cohort. This suggests that this animal did not die due to blood loss and additional circumstances such as heart arrhythmia may have contributed. Animals treated with albumin as an oncotic non-hemostatic protein control in general did not stop bleeding or experienced rebleeding. This suggests that in 
the femoral artery injury model, there may be a clot strength that can be attained by both PolySTAT-4 and -8 , but more slowly by PolySTAT-4, that promotes survival. Since PolySTAT assists most dramatically with retaining clot strength by decreasing fibrinolysis, other models of trauma that are less prone to rebleeding and require faster clotting may provide greater insight to the feasibility of PolySTAT-4 in comparison to variants with higher valency.

While PolySTAT-4 and -8 both performed well in the femoral injury model, there are subtle differences between the two and PolySTAT-16 that require further investigation prior to translation. Notably, decreasing peptide valency decreases the overall molecular weight of the material and may therefore lead to an increased rate of clearance and altered biodistribution. If, in future work this rate is found to be too great, the polymer backbone molecular weight can be increased to counteract lost mass with decreased peptide valency. Despite these differences, to date only one animal treated with a formulation of PolySTAT in the femoral artery injury model has died, resulting in $93 \%$ survival, compared to all control animals (scrambled peptide, albumin, hFXIIIa, and saline) ranging from $0 \%$ to $40 \%$ survival, with an aggregate $20 \%$ survival. Additionally, some insight into the potential mechanism for how PolySTAT interacts with the fibrin has been provided through coagulation assays and neutron scattering experiments. Neutron scattering has also been used to observe structure changes in fibrin during rheological experiments. $^{21}$ This versatility is promising as a tool for gaining additional understanding of the hemostatic mechanisms in general.

\section{Conclusions}

PolySTAT polymers containing 4 or more peptides per polymer on average were effective to restore clot formation and improve survival in a rat bleeding model. The threshold observed in these studies may be due to a minimal average distance between cross-links for 
multivalency and effective clot strengthening, or, as shown by neutron scattering, a minimum valency necessary for additional recruitment of fibrin. Synthetic fibrin-targeting hemostats have great potential to significantly improve survival from traumatic bleeding.

\section{Acknowledgments}

This work was supported by NIH R21EB018637. RJL was supported by an NSF Graduate Fellowship (2013163249) and NIH Training Grant NIBIB T32EB1650. NJW was supported by Grant Number KL2 TR000421 from the National Center for Advancing Translational Sciences (NCATS), a component of the National Institutes of Health (NIH). Its contents are solely the responsibility of the authors and do not necessarily represent the official view of NCATS or NIH. We acknowledge the support of the National Institute of Standards and Technology, U.S. Department of Commerce, in providing the neutron research facilities used in this work and invitational travel support for RJL. This work utilized facilities supported in part by the National Science Foundation under Agreement No. DMR-1508249. We thank James Carothers for use of his Nanodrop 2000. We thank Dr. Xu Wang, and Dr. Susan Stern of the Emergency Medicine Research Laboratory for their technical assistance and expertise. We thank Dr. Leslie Chan for contributing her time and expertise to the PolySTAT project. We thank Dr. Yilong Cheng and Gary Liu for advice and assistance with RBC hemolysis.

\section{References}

1. Kauvar, D. S., Lefering, R. \& Wade, C. E. Impact of Hemorrhage on Trauma Outcome : An Overview of Epidemiology, Clinical Presentations, and Therapeutic Considerations. $J$. Trauma 60, 3-11 (2006).

2. Sauaia, A. et al. Epidemiology of Trauma Deaths: A Reassessment. J. Trauma 38, 185- 
$193(1995)$.

3. Brohi, K., Singh, J., Heron, M. \& Coats, T. Acute traumatic coagulopathy. J. Trauma 54, $1127-1130$ (2003).

4. Raza, I. et al. The incidence and magnitude of fibrinolytic activation in trauma patients. $J$. Thromb. Haemost. 11, 307-314 (2013).

5. Maegele, M. et al. Early coagulopathy in multiple injury: An analysis from the German Trauma Registry on 8724 patients. Injury 38, 298-304 (2007).

6. Niles, S. E. et al. Increased Mortality Associated With the Early Coagulopathy of Trauma in Combat Casualties. J. Trauma Inj. Infect. Crit. Care 64, 1459-1465 (2008).

7. 6 Factor VIII Concentrates, Factor VIII/von Willebrand Factor Concentrates, Factor IX Concentrates, Activated Prothrombin Complex Concentrates. Transfus. Med. Hemotherapy 36, 409-418 (2009).

8. Bertram, J. P. et al. Intravenous Hemostat: Nanotechnology to Halt Bleeding. Sci. Transl. Med. 1, 11-22 (2009).

9. Shoffstall, A. J. et al. Intravenous hemostatic nanoparticles increase survival following blunt trauma injury. Biomacromolecules 13, 3850-3857 (2012).

10. Hubbard, W. B., Lashof-Sullivan, M. M., Lavik, E. B. \& Vandevord, P. J. Steroid-loaded hemostatic nanoparticles combat lung injury after blast trauma. ACS Macro Lett. 4, 387$391(2015)$.

11. Okamura, Y. et al. Development of fibrinogen c-chain peptide-coated, adenosine diphosphate-encapsulated liposomes as a synthetic platelet substitute. J Thromb Haemost 7, 470-477 (2009).

12. Modery-Pawlowski, C. L., Tian, L. L., Ravikumar, M., Wong, T. L. \& Gupta, A. Sen. In 
vitro and in vivo hemostatic capabilities of a functionally integrated platelet-mimetic liposomal nanoconstruct. Biomaterials 34, 3031-3041 (2013).

13. Brown, A. C. et al. Ultrasoft microgels displaying emergent platelet-like behaviours. Nat. Mater. 13, 1-7 (2014).

14. Kolodziej, A. F. et al. Fibrin Specific Peptides Derived by Phage Display: Characterization of Peptides and Conjugates for Imaging. Bioconjug. Chem. 23, 548-556 (2012).

15. Chan, L. W. et al. A synthetic fibrin cross-linking polymer for modulating clot properties and inducing hemostasis. Sci. Transl. Med. 7, (2015).

16. Chan, L. W., White, N. J. \& Pun, S. H. A Fibrin Cross-linking Polymer Enhances Clot Formation Similar to Factor Concentrates and Tranexamic Acid in an in Vitro Model of Coagulopathy. (2016). doi:10.1021/acsbiomaterials.5b00536

17. Fasting, C. et al. Multivalency as a Chemical Organization and Action Principle. Angew. Rev. 51, 10472-10498 (2012).

18. Spain, S. G. \& Cameron, N. R. A spoonful of sugar : the application of glycopolymers in therapeutics. Polym. Chem. 2, 60-68 (2011).

19. Shoffstall, A. J. et al. Tuning ligand density on intravenous hemostatic nanoparticles dramatically increases survival following blunt trauma. Biomacromolecules 14, 27902797 (2013).

20. Weigandt, K. M., Pozzo, D. C. \& Porcar, L. Structure of high density fibrin networks probed with neutron scattering and rheology. Soft Matter 5, 4321-4330 (2009).

21. Weigandt, K. M., Porcar, L. \& Pozzo, D. C. In situ neutron scattering study of structural transitions in fibrin networks under shear deformation. Soft Matter 7, 9992-10000 (2011). 
22. Yanjarappa, M. J., Gujraty, K. V, Joshi, A., Saraph, A. \& Kane, R. S. Synthesis of Copolymers Containing an Active Ester of Methacrylic Acid by RAFT : Controlled Molecular Weight Scaffolds for Biofunctionalization. Biomacromolecules 7, 1665-1670 (2006).

23. Kirby, N. M. et al. research papers A low-background-intensity focusing small-angle Xray scattering undulator beamline. 1670-1680 (2013). doi:10.1107/S002188981302774X

24. Hammouda, B., Ho, D. L. \& Kline, S. Insight into Clustering in Poly ( ethylene oxide ) Solutions. Macromolecules 37, 6932-6937 (2004).

25. Glinka, C. J. et al. The $30 \mathrm{~m}$ Small-Angle Neutron Scattering Instruments at the National Institute of Standards and Technology. J. Appl. Crystallogr. 31, 430-445 (1998).

26. Kline, S. R. Reduction and analysis of SANS and USANS data using IGOR Pro. J. Appl. Crystallogr. 39, 895-900 (2006).

27. Barker, J. G. et al. Design and performance of a thermal-neutron double-crystal diffractometer for USANS at NIST. J. Appl. Crystallogr. 38, 1004-1011 (2005).

28. Terech, P. Structural Study of Cholesteryl Anthraquinone-2-carboxylate (CAQ) Physical Organogels by Neutron and X-ray Small Angle Scattering. J. Phys. Chem. 100, 37593766 (1996).

29. Teixeira, J. Small-Angle Scattering by Fractal Systems. J. Appl. Crystallogr. 21, 781-785 (1988).

30. Hammouda, B. \& Horkay, F. Clustering and Solvation in Poly(acrylic acid) Polyelectrolyte Solutions. Macromolecules 38, 2019-2021 (2005).

31. Dowling, M. B. et al. Biomaterials A self-assembling hydrophobically modified chitosan capable of reversible hemostatic action. Biomaterials 32, 3351-3357 (2011). 
32. Soon, A. S. C., Lee, C. S. \& Barker, T. H. Modulation of fibrin matrix properties via knob:hole affinity interactions using peptide-PEG conjugates. Biomaterials 32, 44064414 (2011).

33. Laudano, A. P. \& Doolittle, R. F. Studies on synthetic peptides that bind to fibrinogen and prevent fibrin polymerization. Structural requirements, number of binding sites, and species differences. Biochemistry 19, 1013-1019 (1980). 